\title{
A Novel Patient-Specific Model for Predicting Postoperative SIRS in Elderly Patients: A Retrospective Cohort Study
}

\section{Chaojin Chen}

Third Affiliated Hospital of Sun Yat-Sen University https://orcid.org/0000-0001-5101-4101

\section{Tongsen Luo}

Third Affiliated Hospital of Sun Yat-Sen University

\section{Xiaoyue Li}

Third Affiliated Hospital of Sun Yat-Sen University

\section{Yaxin Lu}

Third Affiliated Hospital of Sun Yat-Sen University

\section{Jingjing Chen}

Third Affiliated Hospital of Sun Yat-Sen University

\section{Qi Zhang}

Third Affiliated Hospital of Sun Yat-Sen University

\section{Zifeng Liu}

Third Affiliated Hospital of Sun Yat-Sen University

\section{Shaoli Zhou}

Third Affiliated Hospital of Sun Yat-Sen University

\section{Ziqing Hei ( $\square$ heiziqing@sina.com )}

Third Affiliated Hospital of Sun Yat-Sen University https://orcid.org/0000-0001-7466-2769

\section{Research}

Keywords: Nomogram, Postoperative SIRS, Elderly patients, Predicting model

Posted Date: May 17th, 2021

DOI: https://doi.org/10.21203/rs.3.rs-490948/v1

License: (c) (1) This work is licensed under a Creative Commons Attribution 4.0 International License. Read Full License 


\section{Abstract \\ Background}

Postoperative systemic inflammatory response syndrome (SIRS) is common in surgical ICU patients especially in elderly patients, and the geriatric population with SIRS is more susceptible to sepsis, MODS, and death. There have been no reliable and accurate methods to predict SIRS in the elderly. Therefore, we aimed to develop and validate a novel effective model for predicting postoperative SIRS in elderly patients.

\section{Methods}

Data of 16141 patients aged $\geq 65$ years who underwent surgery in two centers of Third Affiliated Hospital of Sun Yat-sen University from January 2015 to September 2020 were retrieved and analyzed. We developed a predictive model for postoperative SIRS based on a retrospectively cohort study of 5904 patients spanning from January 2015 to December 2019. The discriminative performance of this model was determined by area under the receiver operating characteristics curve (AUC). We also used the model to validate outcomes in 1105 patients who underwent surgery at the same institutions from January 2020 to September 2020, and to perform a stratified analysis.

\section{Results}

A total of 5904 patients were enrolled in the training cohort and 1105 patients comprised the validation cohort, in which incidence rates of postoperative SIRS were $24.6 \%$ and $20.2 \%$, respectively. Patients with SIRS had more postoperative complications and a worse in-hospital survival rate than patients without SIRS. Six feature variables including preoperative fever, preoperative serum albumin level, ASA classification, total intraoperative infusion volume, surgical duration, and postoperative intensive care unit admission were identified as valuable predictors to construct the nomogram, with high AUCs in both the training and validation cohorts $(0.800[0.787,0.813]$ and $0.823[0.791,0.855]$, respectively).

\section{Conclusions}

We constructed an effective nomogram using 6 routinely obtained variables to assist clinicians to predict postoperative SIRS in elderly patients.

\section{Background}

Systemic inflammatory response syndrome (SIRS) occurs in one-third of all hospitalized patients and in more than $50 \%$ of all intensive care unit (ICU) patients. Furthermore, over $80 \%$ of surgical ICU patients develop postoperative SIRS(1) and are at considerable risk for severe complications and death. 
Approximately one-third of SIRS patients develop severe sepsis and septic shock (1), causing complications that include multiple organ dysfunction syndrome (MODS) and increased postoperative mortality $(2,3)$. Notably, the incidence of SIRS is significantly higher in patients older than 75 years than in those younger than 40 years of age (4), and the geriatric population with SIRS is more susceptible to sepsis, MODS, and death due to various age-related organ dysfunctions, preexisting comorbidities, and limited physiologic reserve to cope with SIRS-related hemodynamic and metabolic changes(5). The early diagnosis of sepsis is critical because each one-hour delay in treatment increases mortality by $7.6 \%(6)$. Because the consensus definition of severe sepsis requires signs that meet criteria for SIRS (7), the proactive prediction and early diagnosis of postoperative SIRS in elderly patients may enable clinicians to provide timely treatment to prevent sepsis and organ failure.

Multiple risk factors have been identified to facilitate the prediction of postoperative SIRS. These include preoperative high-sensitive C-reactive protein/albumin ratio, C-reactive protein level, erythrocyte sedimentation rate, and platelet-to-lymphocyte ratio(8-10). A nomogram for the prediction of SIRS after transrectal ultrasound-guided prostate biopsy has also been developed (11). However, most of these factors focus on a single organ system or solitary event, or are established from clinical trials not designed to study the geriatric population. An effective risk stratification and prediction model for postoperative SIRS in elderly surgical patients has not yet been available.

The goal of our study was to develop and externally validate an accurate but simple prediction model for postoperative SIRS in elderly patients. We aimed to use routinely measured perioperative variables to create a predictive model that could be easily implemented in clinical practice to support clinicians in the prevention of severe sepsis, septic shock, and postoperative morbidity in elderly patients.

\section{Methods}

\section{Patients}

In this retrospective study, data of 16141 patients aged $\geq 65$ years who underwent surgery in two centers of the Third Affiliated Hospital of Sun Yat-sen University (Guangzhou, Guangdong, China) from January 2015 to September 2020 were retrieved from the Electronic Health Record (EHR) systems. During the retrospective enrollment, patients with preoperative SIRS; those who underwent topical, local, nerve block, or combined spinal epidural anesthesia; and those whose total intraoperative infusion volumes, fluid losses, or ASA classifications were not recorded were excluded.

In the EHR systems of our hospital, a database platform was established by extracting medical records from the hospital information system (HIS), laboratory information system, and Docare Anesthesia System (2005-2020 Medicalsystem Co. Ltd., Suzhou, China). This platform enabled access to comprehensive data collected during hospital admissions, inpatient stays, and post-hospital follow-up visits that included demographic characteristics, daily documentation, laboratory and imaging results, anesthesia records, and other clinical characteristics. 


\section{Definition of SIRS}

A case definition of SIRS was met when a patient exhibited two of the following four criteria as we reported previously (12) : (1) body temperature $\geq 38^{\circ} \mathrm{C}$ or $<36^{\circ} \mathrm{C}$, (2) heart rate $\geq 90$ beats $/ \mathrm{min}$, (3) respiratory rate $\geq 20$ breaths/min or arterial carbon dioxide tension $<32 \mathrm{mmHg}$ and (4) leucocyte count $\geq$ $12 \times 10^{9} / \mathrm{L}$ or $<4 \times 10^{9} / \mathrm{L}$. The incidence of SIRS within three postoperative days was recorded.

\section{Variable definition}

We used descriptive statistics to characterize patients in the training and validation cohorts, both with and without SIRS (Table 1). Twenty variables were selected from the electronic health records based on previously published literature(13-15) and clinical experience. These included demographic variables such as age and gender; comorbid conditions, including diabetes and hypertension; smoking history; preoperative fever; preoperative laboratory variables; preoperative condition, including intubation before surgery; ASA classification; surgical grade; intraoperative events including total infusion volume, total fluid loss, blood loss, surgical duration, and postoperative ICU admission. For continuous variables, data were placed on a scale of approximate symmetry of distribution using the Yeo-Johnson transformation and were data-centered and normalized. 
Table 1

Patient characteristics of non-SIRS and SIRS groups

\begin{tabular}{|c|c|c|c|c|}
\hline \multirow[t]{3}{*}{ Variables } & \multicolumn{2}{|c|}{ Development Cohort ${ }^{1}$} & \multicolumn{2}{|c|}{ Validation Cohort ${ }^{1}$} \\
\hline & Non-SIRS & SIRS & Non-SIRS & SIRS \\
\hline & $4453(75.4)$ & $1451(24.6)$ & $882(79.8)$ & $223(20.2)$ \\
\hline $\operatorname{Age}^{2^{*}}, y$ & $\begin{array}{l}70.0 \\
{[67.0,75.0]}\end{array}$ & $\begin{array}{l}71.0 \\
{[67.0,76.0]}\end{array}$ & $\begin{array}{l}70.0 \\
{[67.0,74.0]}\end{array}$ & $\begin{array}{l}71.0 \\
{[67.0,75.5]}\end{array}$ \\
\hline \multicolumn{5}{|l|}{ Gender ${ }^{1 *}$} \\
\hline Female & $2034(45.7)$ & $542(37.4)$ & $358(40.6)$ & $87(39.0)$ \\
\hline Male & $2419(54.3)$ & $909(62.6)$ & $524(59.4)$ & $136(61.0)$ \\
\hline \multicolumn{5}{|c|}{ Preoperative intubation $^{1 *}$} \\
\hline No & $4388(98.5)$ & $1282(88.4)$ & $878(99.5)$ & $203(91.0)$ \\
\hline Yes & $65(1.46)$ & $169(11.6)$ & $4(0.45)$ & $20(8.97)$ \\
\hline \multicolumn{5}{|c|}{ Hypertension ${ }^{1 \#}$} \\
\hline No & $1756(39.4)$ & $590(40.7)$ & $436(49.4)$ & $114(51.1)$ \\
\hline Yes & $2697(60.6)$ & $861(59.3)$ & $446(50.6)$ & 109 (48.9) \\
\hline \multicolumn{5}{|c|}{ Diabetes mellitus ${ }^{1^{*}}$} \\
\hline No & $2997(67.3)$ & $860(59.3)$ & $823(93.3)$ & $198(88.8)$ \\
\hline Yes & $1456(32.7)$ & $591(40.7)$ & $59(6.69)$ & $25(11.2)$ \\
\hline \multicolumn{5}{|c|}{ History of smoking ${ }^{1^{*}}$} \\
\hline No & 3927 (88.2) & $1215(83.7)$ & $850(96.4)$ & $215(96.4)$ \\
\hline Yes & $526(11.8)$ & $236(16.3)$ & $32(3.63)$ & $8(3.59)$ \\
\hline \multicolumn{5}{|c|}{ ASA classification $^{1 *}$} \\
\hline I/II & $3204(72.0)$ & $663(45.7)$ & $608(68.9)$ & $83(37.2)$ \\
\hline III/IV/V & $1249(28.0)$ & $788(54.3)$ & $274(31.1)$ & $140(62.8)$ \\
\hline \multicolumn{5}{|c|}{ Preoperative fever ${ }^{1 *}$} \\
\hline No & 3971 (89.2) & 1067 (73.5) & $870(98.6)$ & $212(95.1)$ \\
\hline
\end{tabular}

${ }^{1}$ expessed as $\mathrm{n}(\%) ;{ }^{2}$ expessed as median [Q1, Q3]; ${ }^{*}$ Non-SIRS group $v s$. SIRS group in the development cohort, $p<0.001$. WBC count, White blood cell count; ALT, Alanine aminotransferase; hsCRP, high-sensitivity c-reaction protien; SIRS, systemic inflammatory response syndrome; 


\begin{tabular}{|c|c|c|c|c|}
\hline \multirow[t]{3}{*}{ Variables } & \multicolumn{2}{|c|}{ Development Cohort ${ }^{1}$} & \multicolumn{2}{|c|}{ Validation Cohort $^{1}$} \\
\hline & Non-SIRS & SIRS & Non-SIRS & SIRS \\
\hline & $4453(75.4)$ & $1451(24.6)$ & $882(79.8)$ & $223(20.2)$ \\
\hline Yes & $482(10.8)$ & $384(26.5)$ & $12(1.36)$ & $11(4.93)$ \\
\hline \multicolumn{5}{|l|}{ ICU admission $1^{*}$} \\
\hline No & $4326(97.1)$ & 1005 (69.3) & $863(97.8)$ & $132(59.2)$ \\
\hline Yes & $127(2.85)$ & $446(30.7)$ & $19(2.15)$ & $91(40.8)$ \\
\hline \multicolumn{5}{|l|}{ Operation grade ${ }^{1 *}$} \\
\hline I/II & 311 (6.98) & 55 (3.79) & $57(6.46)$ & $10(4.48)$ \\
\hline III & $1672(37.5)$ & 309 (21.3) & $311(35.3)$ & $33(14.8)$ \\
\hline IV & $2470(55.5)$ & $1087(74.9)$ & $514(58.3)$ & $180(80.7)$ \\
\hline $\mathrm{WBC}^{2^{\star}}, \times 10^{\wedge} 9 / \mathrm{L}$ & $\begin{array}{l}6.34 \\
{[5.17,7.91]}\end{array}$ & $\begin{array}{l}7.04 \\
{[5.47,9.32]}\end{array}$ & $\begin{array}{l}6.46 \\
{[5.16,8.08]}\end{array}$ & $\begin{array}{l}7.66 \\
{[5.95,11.4]}\end{array}$ \\
\hline $\begin{array}{l}\text { Total volume of infusion }{ }^{2} \text {, } \\
\mathrm{mL}\end{array}$ & $\begin{array}{l}1500 \\
{[1000,2200]}\end{array}$ & $\begin{array}{l}2200 \\
{[1500,3200]}\end{array}$ & $\begin{array}{l}1500 \\
{[1000,2200]}\end{array}$ & $\begin{array}{l}2112 \\
{[1512,3050]}\end{array}$ \\
\hline $\mathrm{ALT}^{2 *}, \mathrm{U} / \mathrm{L}$ & $\begin{array}{l}17.0 \\
{[13.0,25.0]}\end{array}$ & $\begin{array}{l}19.0 \\
{[13.0,30.0]}\end{array}$ & $\begin{array}{l}17.0 \\
{[13.0,25.0]}\end{array}$ & $\begin{array}{l}17.0 \\
{[12.8,28.0]}\end{array}$ \\
\hline hs-CRP ${ }^{2 *}, \mathrm{mg} / \mathrm{L}$ & $\begin{array}{l}6.34 \\
{[5.11,7.97]}\end{array}$ & $\begin{array}{l}7.10 \\
{[5.47,9.60]}\end{array}$ & $\begin{array}{l}6.50 \\
{[5.10,8.31]}\end{array}$ & $\begin{array}{l}7.80 \\
{[6.01,11.8]}\end{array}$ \\
\hline Albumin $^{2 *}, \mathrm{~g} / \mathrm{L}$ & $\begin{array}{l}40.3 \\
{[37.2,43.2]}\end{array}$ & $\begin{array}{l}38.2 \\
{[34.6,41.5]}\end{array}$ & $\begin{array}{l}39.9 \\
{[36.3,42.9]}\end{array}$ & $\begin{array}{l}37.8 \\
{[33.0,41.5]}\end{array}$ \\
\hline Creatinine ${ }^{2^{*}}, \mu \mathrm{mol} / \mathrm{L}$ & $\begin{array}{l}74.0 \\
{[61.0,89.0]}\end{array}$ & $\begin{array}{l}78.0 \\
{[63.0,96.0]}\end{array}$ & $\begin{array}{l}71.0 \\
{[60.0,86.0]}\end{array}$ & $\begin{array}{l}74.0 \\
{[56.0,98.5]}\end{array}$ \\
\hline Duration of surgery ${ }^{2^{\star}}, \min$ & $125[75.0,200]$ & $192[120,295]$ & $131[77.0,215]$ & $193[120,302]$ \\
\hline $\begin{array}{l}\text { Total volume of fluid, } \\
\text { loss }^{2^{*}}, \mathrm{~mL}\end{array}$ & $350[110,700]$ & $680[350,1200]$ & $400[100,750]$ & $800[350,1125]$ \\
\hline Blood loss $2^{2 *}, \mathrm{~mL}$ & $50.0[10.0,100]$ & $100[50.0,200]$ & $40.0[10.0,100]$ & $100[50.0,200]$ \\
\hline Hemoglobin ${ }^{2 *}, \mathrm{~g} / \mathrm{L}$ & $128[116,139]$ & $124[108,137]$ & $126[115,139]$ & $116[100,134]$ \\
\hline
\end{tabular}

\section{Variable selection}


For univariate selection, we used a resampling technique with 10-fold cross-validation, to consider two logistic regression models for each variable: a null model containing only the intercept term, and a complex model with a single individual predictor in the risk set. We calculated the area under the receiver operating characteristic (ROC) curve in the two different models and performed a one-sided t-test to determine the difference between the two area under the receiver operating characteristics (AUC) values(16). The AUC value was calculated to determine the importance of each predictor.

\section{Development of risk prediction model and external validation}

In reference to the univariate selection results, we used a combination of Brute Force algorithms (which considered the predictive effect of each combination of candidate variables for outcomes) and clinical guidance, and finally chose 6 variables to comprise our final model. The Hosmer-Lemeshow test was used to assess the model's goodness of fit. Using the Irm function of the RMS package, we constructed a nomogram to facilitate clinical decision-making. In this plot, each patient's score for each variable axis can be determined by plotting a line upward. The sum of these individual scores is plotted as a line down the total score axis to the risk axis, which predicts the likelihood of SIRS occurring in that patient. To perform external validation, we calibrated the intercept term $\beta$ of the logistic with probabilities and recalculated the probabilities of the external validation set after calibration. All statistical analyses were performed by using R version 3.6.2 software (Institute for Statistics and Mathematics, Vienna, Austria; http://www.r-project.org)(17).

\section{Results}

A total of 16141 patients aged $\geq 65$ years spanning the period from January 2015 to September 2020 were included. As shown in Fig. 1, 533 patients with preoperative SIRS, 7835 patients receiving regional anesthesia or general anesthesia without intubation, 76 patients with missing anesthesia data, 650 patients whose total volumes of infusion or fluid loss were 0 , and 35 patients with missing ASA classifications were excluded. Ultimately, 5904 patients receiving general anesthesia with endotracheal intubation and spanning from January 2015 to December 2019 were enrolled in the training cohort, whereas 1105 patients from January 2020 to September 2020 comprised the validation cohort. The incidence rates of postoperative SIRS in the two cohorts were $24.6 \%$ and $20.2 \%$, respectively.

\section{Characteristics of development and validation cohorts}

The demographic data and clinical characteristics of 7009 cases are displayed in Table S1. Three thousand twenty-one (43.1\%) patients were women, and the average age was 70 (67.0-75.0) years. The numbers of SIRS patients in the development and validation cohorts were 1451 and 882, respectively. The prevalence rates of hypertension, diabetes mellitus, and smoking history were $58.7 \%$ (4113), $30.4 \%$ (2131), and $11.4 \%$ (802) respectively. Eight hundred eighty-nine (12.7\%) patients had preoperative fever 


\section{Differences in characteristics between Non-SIRS and SIRS groups.}

A total of 20 features were collected from each patient in the training cohort (Table 1). After comparing the characteristics of patients with or without postoperative SIRS, we found that patients who developed postoperative SIRS were older (71.0 [67.0,76.0] vs. $70.0[67.0,75.0], p<0.001$, Table 1); more likely to have been assessed at ASA III/IV/V ( $54.3 \%$ vs. $28.0 \%, p<0.001)$; and more likely to have diabetes mellitus, a history of smoking, preoperative fever, and to have undergone preoperative intubation (all $p<0.001$, Table 1). Grade IV surgeries were more frequent in the SIRS group $(74.9 \%$ vs. $55.5 \%, p<0.001)$.

Meanwhile, preoperative leukocyte counts and alanine aminotransferase, hs-CRP, and creatinine levels were higher in SIRS patients than in non-SIRS patients; while levels of hemoglobin and albumin were lower in SIRS patients (all $p<0.001$ ). Moreover, SIRS patients had larger intraoperative infusion, fluid loss, and blood loss volumes; and longer surgical durations than those of non-SIRS patients (all $p<0.001$ ).

\section{Prognosis of Non-SIRS and SIRS groups}

As shown in Tigure 2, compared with the Non-SIRS group, patients in the SIRS group were significantly more likely to experience postoperative complications that included agitation and delirium, hemorrhage, pneumonia, acute kidney injury, hypotension, coma, and cardiac arrest. Patients with SIRS had a significantly worse in-hospital survival rate than patients without SIRS (all $p \leq 0.001$, Table 2). SIRS patients also had higher hospitalization and surgical costs, and longer postoperative and total hospital stays than those without SIRS (all $p<0.001$, Table 2 ). 
Table 2

Patients' postoperative prognosis between the two groups.

\begin{tabular}{|c|c|c|c|c|}
\hline Variables & $\begin{array}{l}\text { Total } \\
(\mathrm{N}=7009)\end{array}$ & $\begin{array}{l}\text { Non-SIRS } \\
5335(76.1)\end{array}$ & $\begin{array}{l}\text { SIRS } \\
1674(23.9)\end{array}$ & $\begin{array}{l}P \\
\text { value }\end{array}$ \\
\hline Hemorrhage $^{1}$ & $2140(30.5)$ & $1394(26.1)$ & $746(44.6)$ & $<_{0.001}^{<}$ \\
\hline ARDS $^{1}$ & $26(0.37)$ & $9(0.17)$ & $17(1.02)$ & $\dot{0} .001$ \\
\hline Pneumonia $^{1}$ & $550(7.85)$ & $169(3.17)$ & $381(22.8)$ & $\dot{0} .001$ \\
\hline $\begin{array}{l}\text { Acute pulmonary } \\
\text { embolism }^{1}\end{array}$ & $15(0.21)$ & $5(0.09)$ & $10(0.60)$ & 0.001 \\
\hline Cardiac arrest $^{1}$ & $91(1.30)$ & $19(0.36)$ & $72(4.30)$ & $\begin{array}{l}< \\
0.001\end{array}$ \\
\hline Hypotension ${ }^{1}$ & $187(2.67)$ & $73(1.37)$ & $114(6.81)$ & $\dot{0} .001$ \\
\hline Agitation and delirium ${ }^{1}$ & $187(2.67)$ & $51(0.96)$ & $136(8.13)$ & $\dot{0} .001$ \\
\hline Coma $^{1}$ & $188(2.68)$ & $18(0.34)$ & $170(10.2)$ & $\begin{array}{l}< \\
0.001\end{array}$ \\
\hline $\begin{array}{l}\text { Mortality during } \\
\text { hospitalization }^{1}\end{array}$ & $70(1.00)$ & $16(0.30)$ & $54(3.23)$ & $\begin{array}{l}<.001 \\
0.00\end{array}$ \\
\hline ICU Admission ${ }^{1}$ & $683(9.74)$ & $146(2.74)$ & $537(32.1)$ & $\dot{0} 001$ \\
\hline Acute kidney injury ${ }^{1}$ & $223(3.18)$ & $93(1.74)$ & $130(7.77)$ & $\dot{0} .001$ \\
\hline $\begin{array}{l}\text { Postoperative hospital } \\
\text { stay }^{2}\end{array}$ & $7.00[5.00,12.0]$ & $7.00[4.00,10.0]$ & $12.0[8.00,20.0]$ & $\dot{0} 001$ \\
\hline Total hospital stay ${ }^{2}$ & $15.0[10.0,22.0]$ & $13.0[9.00,19.0]$ & $21.0[14.0,31.0]$ & $\begin{array}{l}< \\
0.001\end{array}$ \\
\hline Total cost ${ }^{2}$ & $\begin{array}{l}55499 \\
{[29795,83605]}\end{array}$ & $\begin{array}{l}47077 \\
{[25392,71091]}\end{array}$ & $\begin{array}{l}89500 \\
{[59959,136333]}\end{array}$ & $\dot{0} .001$ \\
\hline Costs of surgery ${ }^{2}$ & $5780[3679,8389]$ & $5300[3340,7902]$ & $7230[4877,9816]$ & $\hat{0}_{0.001}$ \\
\hline
\end{tabular}


Univariate analysis of patient risk factors for perioperative SIRS was performed by using a resampling technique with 10-fold cross-validation. Results are shown in Table S2. The univariate association between each predictor and SIRS is represented by box chart, and percentages are shown in the stacked bar chart in Figure S1. Based on the results of resampling methods, previous literature reports, and clinical experience, we selected 6 feature variables that had strong independent discriminatory power for SIRS with maximal AUC to construct the logistic regression model. These included preoperative fever, preoperative albumin level, ASA classification, total intraoperative infusion volume, surgical duration, and postoperative ICU admission.

\section{Model construction and external validation}

In the training cohort, the logistic regression model established by using the 6 selected predictors had a high AUC $(0.800$ [ $95 \% \mathrm{Cl}, 0.787-0.813])$ to discriminate individuals with SIRS from those with non-SIRS, with a sensitivity of $71.8 \%$ and specificity of $71.8 \%$ (Fig. 2A, Table S3).

To better confirm the results of the logistic regression model, we then used the developed training model for external validation of another cohort of 1105 patients. The result of external validation showed that the AUC of the model was $0.823(95 \% \mathrm{Cl}, 0.791,0.855)$, with a sensitivity of $74.0 \%$ and specificity of $74.0 \%$ (Fig. 2B, Table S3). The calibration curve used to predict the presence or absence of SIRS is shown in Fig. 2C (Calibrated Hosmer-Lemeshow test, $\mathrm{p}=0.01$ ).

In addition, we also used the six-variable model to analyze the validation cohort hierarchically based on the potential confounding factors of SIRS. We found no significant differences between subgroups of age, gender, diabetes mellitus, hypertension, blood loss and operation grade (Table 3 ), indicating that the predictive performance of the developed model in each subgroup was relatively stable, and illustrating its high-accuracy and generalizability in each subgroup. 
Table 3

Model effects of stratification analysis in the validation set

\begin{tabular}{|llll|}
\hline Characteristics & $\mathbf{N}(\%)$ & AUC & $95 \% \mathrm{Cl}$ \\
\hline Total set & 1105 & 0.823 & $0.791-0.855$ \\
\hline Age (years) & & & \\
\hline $65-75$ & $833(75.4)$ & 0.830 & $0.792-0.868$ \\
\hline$\geq 75$ & $272(24.6)$ & 0.787 & $0.725-0.849$ \\
\hline Gender & & & \\
\hline Female & $445(40.27)$ & 0.851 & $0.803-0.899$ \\
\hline Male & $660(59.73)$ & 0.804 & $0.762-0.847$ \\
\hline Diabetes mellitus & & & \\
\hline No & $1021(92.40)$ & 0.825 & $0.791-0.859$ \\
\hline Yes & $84(7.60)$ & 0.781 & $0.671-0.891$ \\
\hline Operation grade & & & \\
\hline I/II & $67(6.06)$ & 0.905 & $0.830-0.981$ \\
\hline III & $344(31.13)$ & 0.803 & $0.725-0.881$ \\
\hline IV & $694(62.81)$ & 0.802 & $0.762-0.842$ \\
\hline Hypertension & & & \\
\hline No & $550(49.77)$ & 0.828 & $0.784-0.873$ \\
\hline Yes & $555(50.23)$ & 0.817 & $0.771-0.862$ \\
\hline Blood loss (mL) & & & \\
\hline$<100$ & & 0.816 & $0.769-0.863$ \\
\hline$\geq 100$ & & 0.772 & $0.719-0.825$ \\
\hline
\end{tabular}

\section{Predictive Nomogram}

Based on the final regression analysis, a nomogram was constructed that incorporated the 6 significant risk factors for predicting postoperative SIRS (Fig. 3). As reported previously (18), each variable corresponding to the nomogram was scored on a point scale axis based on its contribution to our logistic regression model. The total points that corresponded to the risk of postoperative SIRS could be calculated easily that corresponded to the risk of postoperative SIRS could be calculated easily by adding each single score. An online risk calculator to further facilitate external validation can be visited at http://wb.aidcloud.cn/zssy/SIRS.html(19). 


\section{Discussion}

Because of the continuum between different stages of the inflammatory response from SIRS to sepsis and septic shock (20), early diagnosis of postoperative SIRS is critical to initiate timely interventions to prevent septic shock and improve clinical outcomes in elderly patients. Heretofore, the early prediction of postoperative SIRS has been challenging, and there have been no reliable and accurate methods to predict SIRS in the elderly. In this study, we identified 6 feature variables that have strong independent discriminatory power for SIRS with maximal AUC values. Furthermore, we constructed a nomogram composed of the 6 features that had high sensitivity and specificity to distinguish elderly patients at high risk for postoperative SIRS and to alert clinicians to provide early interventions.

Our novel nomogram has important implications for public health policy, clinical practice, and the informed consent process. Firstly, the early identification of elderly patients at risk of progression to postoperative SIRS and the generation of an individualized probability for each patient can be achieved by using our validated prediction tools. Implementation will potentially lead to better management and optimal use of medical resources, and will be critical to improve the survival rates of high-risk patients, especially those in surgical ICUs. Secondly, all of the variables integrated in the predictive model are measured routinely during the perioperative management for elderly surgical patients in China where both the elective patients and emergency patients admitted to the hospital have value of preoperative Albumin. To further facilitate its external validation and application, we have established an online risk calculator (http://wb.aidcloud.cn/zssy/SIRS.html)(19) and it has been accessible for all the peers in daily clinical practice. Moreover, the model can also be easily incorporated into EHR and HIS systems, thus having straightforward applicability, and thus enabling the integration of a risk prediction tool as a clinical decision support aid in perioperative elderly patient care(21). Additionally, our nomogram can be used as a decision support tool in the informed consent process by providing adequate information regarding risks and benefits defined by using a personalized medicine approach to estimate the individualized probability of postoperative SIRS.(22).

To our knowledge, this was the first study to develop a model and construct a quantitative nomogram to predict the probability of postoperative SIRS in aged patients. Because of the critical significance of the early prediction of postoperative SIRS, the identification of predisposing factors is of crucial importance. Several risk factors have been associated with postoperative SIRS including mannose-binding lectin deficiency(23), high levels of circulating GM-CSF + CD4 + T cells(24), bacteriuria and renal stone size (25), diabetes mellitus, and the intraoperative use of an intra-aortic balloon pump(26). However, their predictive values are limited because measurements of most of these parameters are generally not available or easily obtainable in routine testing, or only pertain to particular surgical operations. These limitations preclude their general application to the geriatric population. Fernando et al. developed a model using a Bayesian network approach to predict SIRS in patients admitted to ICU with acute infections (27). In comparison, a nomogram could present a quantitative and practical prediction tool for risk stratification for patients at risk for postoperative SIRS, with the ability to generate a patient-specific numerical probability of a clinical event by integrating diverse prognostic and determinant variables $(28,29)$. 
Wang et al. developed a nomogram to predict SIRS after transrectal ultrasound-guided prostate biopsy (11). However, it was evaluated in a single-center study with a small sample size and without external validation. In the current study, we enrolled 16141 patients aged $\geq 65$ years from two medical centers and divided the patients into training and validation cohorts. We trained the logistic regression model on 19 statistically significant features and incorporated 6 comprehensive and easily accessible variables to form our nomogram, which performed well as evidenced by the AUCs of 0.800 and 0.823 in the training and validation cohorts, respectively. Furthermore, the calibration curves of our nomogram demonstrated an agreement between predictions and actual observations.

The 6 easily-accessible features incorporated into our novel predictive model included preoperative fever, preoperative albumin level, ASA classification, intraoperative total infusion volume, surgical duration, and postoperative ICU admission; most of which have been associated with SIRS (30-35). All of the variables were chosen based on the results of resampling methods, previous literature, and clinical experience. Although some of the factors may be very practice dependent, for instance, the total volume of infusion and postoperative ICU admission are strong predictors that heavily weighted in the nomogram, we have tried to validate the utility of this nomogram in another 1105 patients in 2020 and found it performed well in predicting postoperative SIRS and provide clinicians with practical guidelines to facilitate early diagnosis and proactive interventions in elderly patients to avoid worsen complications, especially for the clinicians in surgical ICU and for the aged patient with preoperative fever, a preoperative albumin level < $30 \mathrm{~g} / \mathrm{L}$, an ASA classification of III/IV/V, an intraoperative total infusion volume $>2000 \mathrm{ml}$, a surgical duration of $>200$ min, and who is admitted to ICU after surgery (Fig. 3).

Several limitations in this study should be addressed. Firstly, the retrospective study design may be prone to collection and entry bias, as well as residual confounding. Secondly, we did not report the incidence of sepsis in the study since that the key data needed for sepsis-3 criteria to diagnose sepsis is missing due to the retrospective design. Thirdly, as the elderly patients receiving regional anesthesia in our hospital are generally in relatively good conditions and often require a short and minor operation that might have lower risk of postoperative SIRS, we only enrolled the patients with general anesthesia and endotracheal intubation in the study. Fourthly, despite having high sensitivity and specificity to identify elderly patients at high risk for postoperative SIRS, the model may potentially miss important factors that could not be accounted for in the retrospective analysis such as genetic or clinical factors that might be even more important to predict postoperative SIRS(26). Future prospective studies are needed to collect more clinical and genomic information to predict an individual patient's predisposition to SIRS more precisely.

\section{Conclusions}

In conclusion, we have developed and externally validated an effective model for predicting the risk of postoperative SIRS in elderly patients. Based on the model, we constructed a practical nomogram that is highly accurate, and that exhibits excellent calibration. This nomogram might enable clinicians to make individualized predictions of each patient's probability of postoperative SIRS and to improve treatment recommendations for elderly patients. 


\section{List Of Abbreviations}

SIRS: systemic inflammatory response syndrome; AUC: area under the receiver operating characteristics curve; ICU: intensive care unit; MODS: multiple organ dysfunction syndrome; HER: Electronic Health Record; HIS: hospital information system; ROC: receiver operating characteristic.

\section{Declarations}

\section{Funding}

This study was supported partly by the National Natural Science Foundation of China (Grant No. 81974296 for Prof. Ziqing Hei), Postdoctoral Science Foundation of China (Grant No. 2019M663260; 2020T130148ZX for Dr. Chaojin Chen) and Basic and Applied Basic Research Foundation of Guangdong Province (Grant No. 2019A1515110020 for Dr. Chaojin Chen)

\section{Conflicts of interest}

The authors declare that they have no conflicts of interest.

\section{Availability of data and material}

The raw data supporting the conclusions of this article will be made available by the corresponding authors via email.

\section{Authors' contributions}

Manuscript writing: CC, XL, QZ; Data analysis: ZL, YL, JC; Cases and supplementary search: TL, CC; Project guidance: $\mathrm{ZH}, \mathrm{SZ}, \mathrm{ZL}$.

\section{Ethics approval}

This study was approved by the Institutional Ethics Committee from the two hospitals and was censored on 18 May 2019 (No.[2019]02-609-01). The requirement for informed consent and clinical trial registration were waived by the committee.

\section{Consent to participate}

Not applicable.

\section{Consent for publication}

Not applicable.

\section{Acknowledgments}


The authors thank all of the patients who kindly participated in this study.

\section{References}

1. Brun-Buisson C. The epidemiology of the systemic inflammatory response. Intensive Care Med. 2000;26 Suppl 1(Suppl 1):S64-74.

2. Ferraris VA, Ballert EQ, Mahan A. The relationship between intraoperative blood transfusion and postoperative systemic inflammatory response syndrome. Am J Surg. 2013;205(4):457-65.

3. Singh P, Yadav S, Singh A, Saini AK, Kumar R, Seth A, et al. Systemic Inflammatory Response Syndrome Following Percutaneous Nephrolithotomy: Assessment of Risk Factors and Their Impact on Patient Outcomes. Urol Int. 2016;96(2):207-11.

4. Gokalp O, Yesilkaya NK, Bozok S, Besir Y, Iner H, Durmaz H, et al. Effects of age on systemic inflammatory response syndrome and results of coronary bypass surgery. Cardiovascular Journal of Africa. 2018;29(1):22-5.

5. Koo EG, Lai LM, Choi GY, Chan MT. Systemic inflammation in the elderly. Best Pract Res Clin Anaesthesiol. 2011;25(3):413-25.

6. Castellanos-Ortega A, Suberviola B, García-Astudillo LA, Holanda MS, Ortiz F, Llorca J, et al. Impact of the Surviving Sepsis Campaign protocols on hospital length of stay and mortality in septic shock patients: results of a three-year follow-up quasi-experimental study. Crit Care Med. 2010;38(4):103643.

7. Kaukonen KM, Bailey M, Pilcher D, Cooper DJ, Bellomo R. Systemic inflammatory response syndrome criteria in defining severe sepsis. N Engl J Med. 2015;372(17):1629-38.

8. Xu H, Hu L, Wei X, Niu J, Gao Y, He J, et al. The Predictive Value of Preoperative High-Sensitive CReactive Protein/Albumin Ratio in Systemic Inflammatory Response Syndrome After Percutaneous Nephrolithotomy. J Endourol. 2019;33(1):1-8.

9. Ganesan V, Brown RD, Jiménez JA, De S, Monga M. C-Reactive Protein and Erythrocyte Sedimentation Rate Predict Systemic Inflammatory Response Syndrome After Percutaneous Nephrolithotomy. J Endourol. 2017;31(7):638-44.

10. Cetinkaya M, Buldu I, Kurt O, Inan R. Platelet-to-Lymphocyte Ratio: A New Factor for Predicting Systemic Inflammatory Response Syndrome after Percutaneous Nephrolithotomy. Urol J. 2017;14(5):4089-93.

11. Wang ZM, Yu YM, Liu JM, Feng F, Huang S, Peng WH. [Establishment of a nomogram model to predict systemic inflammatory response syndrome after transrectal ultrasound-guided prostate biopsy]. Zhonghua Nan Ke Xue. 2018;24(12):1089-93.

12. Deng Y, Tan F, Gan X, Li X, Ge M, Gong C, et al. Perioperative application of dexmedetomidine for postoperative systemic inflammatory response syndrome in patients undergoing percutaneous nephrolithotomy lithotripsy: results of a randomised controlled trial. BMJ Open. 2018;8(11):e019008.

13. Sihler KC, Napolitano LM. Complications of massive transfusion. Chest. 2010;137(1):209-20. 
14. Buxbaum JL, Quezada M, Da B, Jani N, Lane C, Mwengela D, et al. Early Aggressive Hydration Hastens Clinical Improvement in Mild Acute Pancreatitis. Am J Gastroenterol. 2017;112(5):797-803.

15. Alazawi W, Pirmadjid N, Lahiri R, Bhattacharya S. Inflammatory and Immune Responses to Surgery and Their Clinical Impact. Ann Surg. 2016;264(1):73-80.

16. Kuhn M, Johnson K. Feature Engineering and Selection2019.

17. Gentleman R, Ihaka R. A language and environment for statistical computing. Computing. 2011;1:1221.

18. Jiang E, Guo H, Yang B, Li P, Mishra P, Yang T, et al. Predicting and comparing postoperative infections in different stratification following PCNL based on nomograms. Sci Rep. 2020;10(1):11337.

19. An online risk calculator [Available from: http://wb.aidcloud.cn/zssy/SIRS.html.

20. Rangel-Frausto MS, Pittet D, Costigan M, Hwang T, Davis CS, Wenzel RP. The natural history of the systemic inflammatory response syndrome (SIRS). A prospective study. Jama. 1995;273(2):117-23.

21. Tangri N, Stevens LA, Griffith J, Tighiouart H, Djurdjev O, Naimark D, et al. A predictive model for progression of chronic kidney disease to kidney failure. JAMA. 2011;305(15):1553-9.

22. Gupta PK, Gupta H, Sundaram A, Kaushik M, Fang X, Miller WJ, et al. Development and validation of a risk calculator for prediction of cardiac risk after surgery. Circulation. 2011;124(4):381-7.

23. Fidler KJ, Wilson P, Davies JC, Turner MW, Peters MJ, Klein NJ. Increased incidence and severity of the systemic inflammatory response syndrome in patients deficient in mannose-binding lectin. Intensive Care Med. 2004;30(7):1438-45.

24. Huang H, Wang S, Jiang T, Fan R, Zhang Z, Mu J, et al. High levels of circulating GM-CSF(+)CD4(+) T cells are predictive of poor outcomes in sepsis patients: a prospective cohort study. Cell Mol Immunol. 2019;16(6):602-10.

25. Kreydin El, Eisner BH. Risk factors for sepsis after percutaneous renal stone surgery. Nat Rev Urol. 2013;10(10):598-605.

26. Dieleman JM, Peelen LM, Coulson TG, Tran L, Reid CM, Smith JA, et al. Age and other perioperative risk factors for postoperative systemic inflammatory response syndrome after cardiac surgery. Br J Anaesth. 2017;119(4):637-44.

27. Zampieri FG, Aguiar FJ, Bozza FA, Salluh JIF, Soares M, Investigators OS. Modulators of systemic inflammatory response syndrome presence in patients admitted to intensive care units with acute infection: a Bayesian network approach. Intensive Care Med. 2019;45(8):1156-8.

28. Balachandran VP, Gonen M, Smith JJ, DeMatteo RP. Nomograms in oncology: more than meets the eye. Lancet Oncol. 2015;16(4):e173-80.

29. Gong J, Ou J, Qiu X, Jie Y, Chen Y, Yuan L, et al. A Tool for Early Prediction of Severe Coronavirus Disease 2019 (COVID-19): A Multicenter Study Using the Risk Nomogram in Wuhan and Guangdong, China. Clinical Infectious Diseases. 2020;71(15):833-40. 
30. Shaw AD, Raghunathan K, Peyerl FW, Munson SH, Paluszkiewicz SM, Schermer CR. Association between intravenous chloride load during resuscitation and in-hospital mortality among patients with SIRS. Intensive Care Med. 2014;40(12):1897-905.

31. Southern JB, Higgins AM, Young AJ, Kost KA, Schreiter BR, Clifton M, et al. Risk Factors for Postoperative Fever and Systemic Inflammatory Response Syndrome After Ureteroscopy for Stone Disease. J Endourol. 2019;33(7):516-22.

32. Fuchs $L$, Chronaki CE, Park S, Novack V, Baumfeld $Y$, Scott $D$, et al. ICU admission characteristics and mortality rates among elderly and very elderly patients. Intensive Care Med. 2012;38(10):1654-61.

33. Hackett NJ, De Oliveira GS, Jain UK, Kim JY. ASA class is a reliable independent predictor of medical complications and mortality following surgery. Int J Surg. 2015;18:184-90.

34. Arnau-Barres I, Guerri-Fernandez R, Luque S, Sorli L, Vazquez O, Miralles R. Serum albumin is a strong predictor of sepsis outcome in elderly patients. Eur J Clin Microbiol Infect Dis. 2019;38(4):743-6.

35. Garami A, Steiner AA, Romanovsky AA. Fever and hypothermia in systemic inflammation. Handb Clin Neurol. 2018;157:565-97.

\section{Figures}




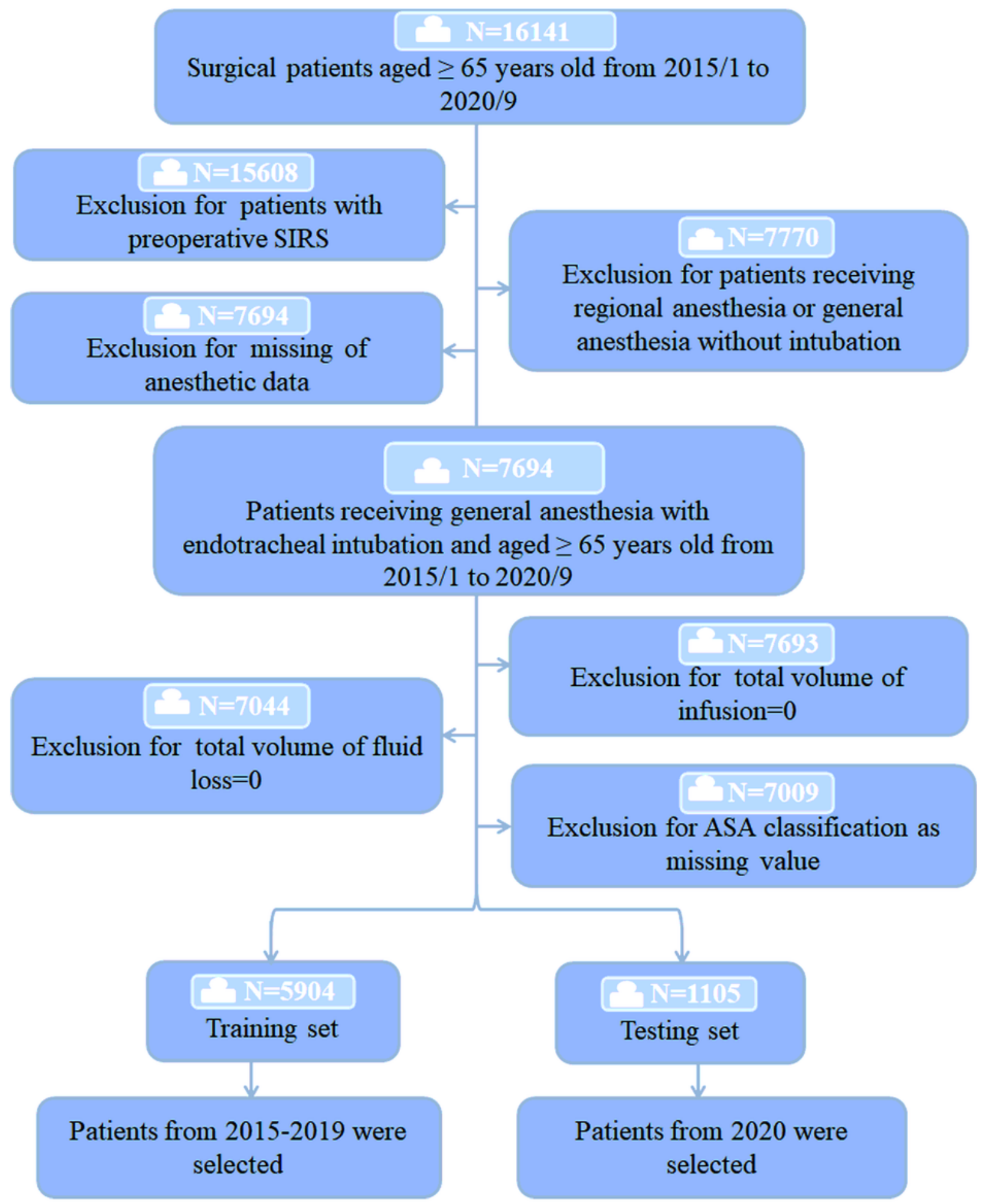

\section{Figure 1}

Flow chart demonstrating the patient selection process 
A

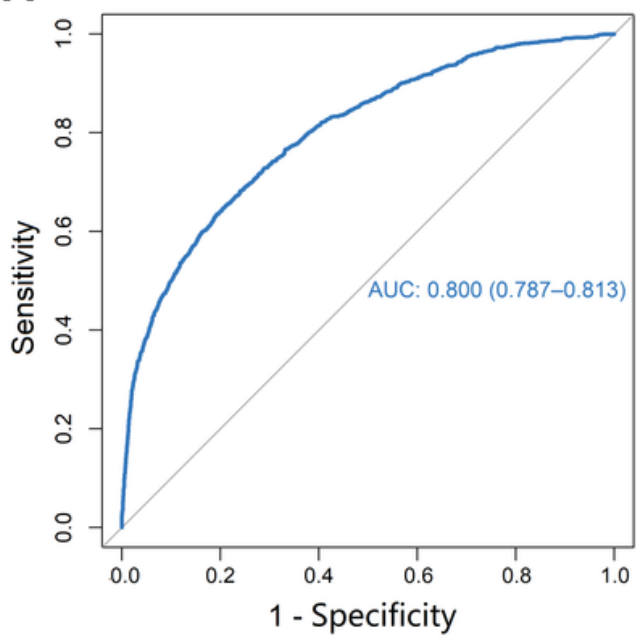

B

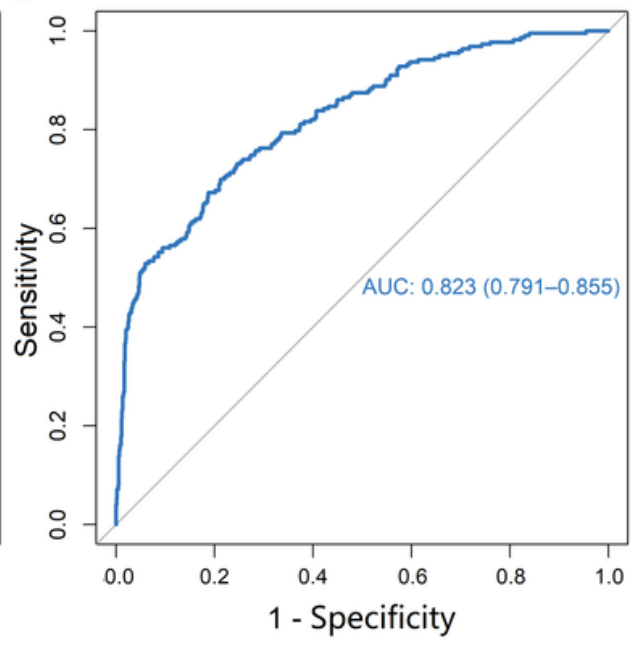

C

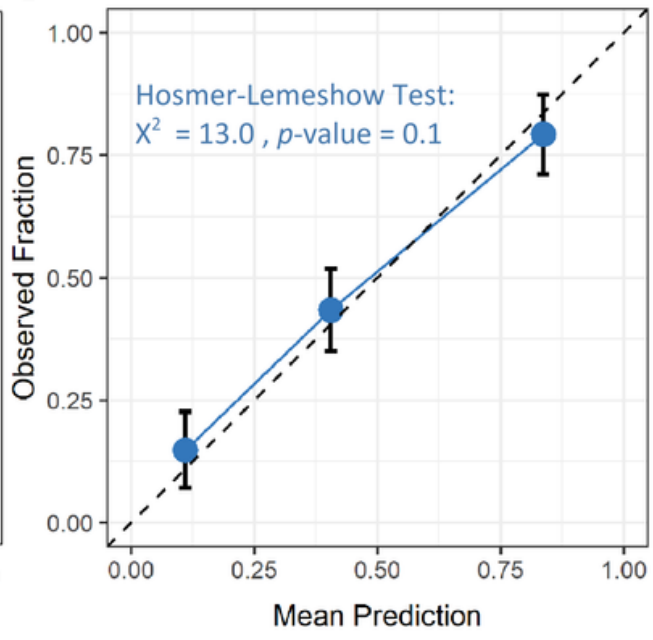

Figure 2

ROC plot and calibration of the logistic regression model ROC plot for development (A) and validation (B) set; (C) The calibration curve for predicting patient SIRS.

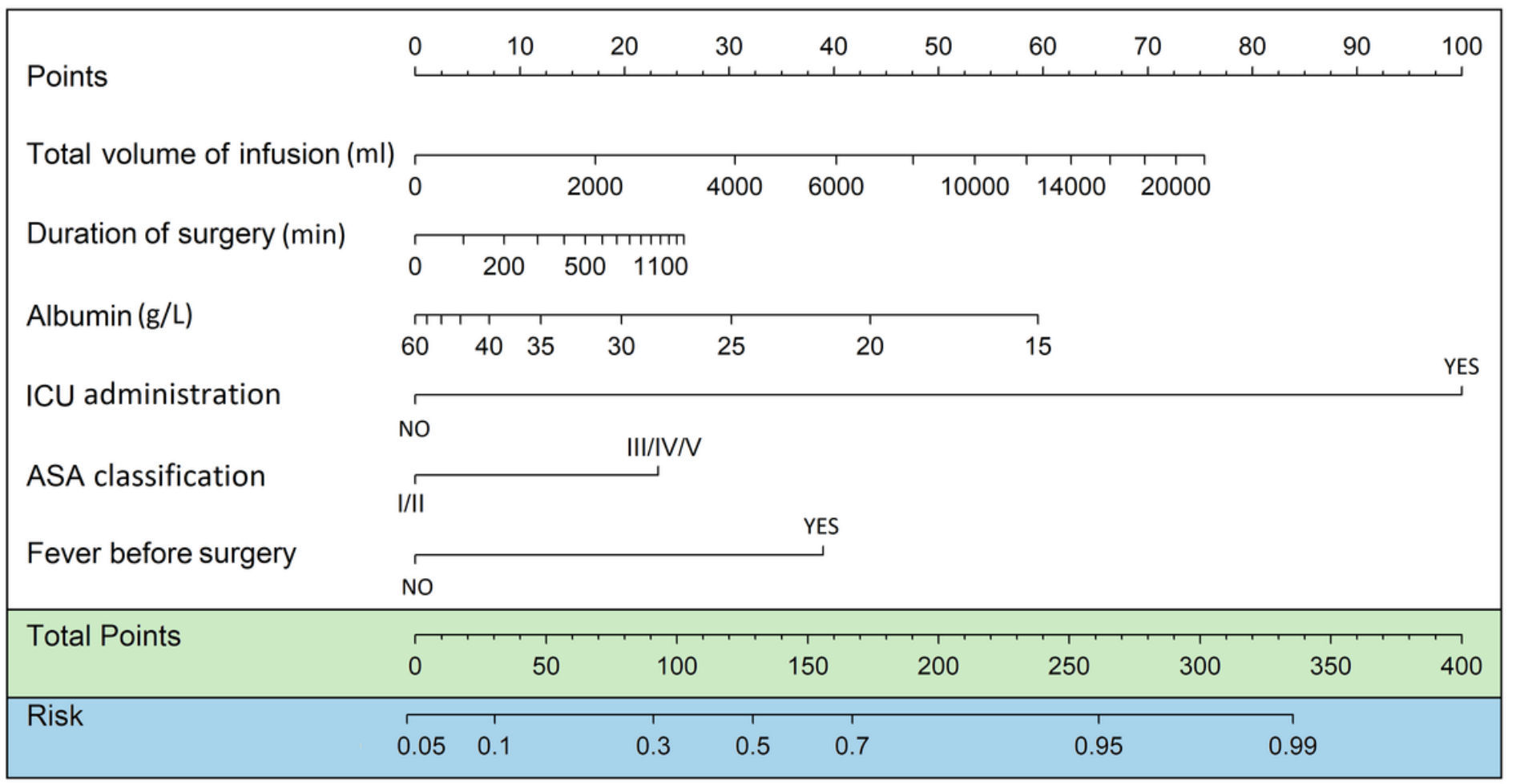

Figure 3

Nomogram to predict postoperative SIRS in elderly patients

\section{Supplementary Files}

This is a list of supplementary files associated with this preprint. Click to download. 
- Supplementarymaterial.docx

Page 20/20 